\title{
Review Article \\ Biologic Effect of Hydrogen Sulfide and Its Role in Traumatic Brain Injury
}

\author{
Jiaxin Zhang $\mathbb{D}$, ${ }^{1}$ Shaoyi Zhang $\mathbb{D},{ }^{1}$ Haiyan Shan $\mathbb{D}^{2}$, and Mingyang Zhang $\mathbb{D}^{1}$ \\ ${ }^{1}$ Institute of Forensic Sciences, School of Biology \& Basic Medical Sciences, Soochow University, Suzhou, China \\ ${ }^{2}$ Department of Obstetrics and Gynecology, The Affiliated Suzhou Hospital of Nanjing Medical University, Suzhou, China
}

Correspondence should be addressed to Haiyan Shan; ghostqth@163.com and Mingyang Zhang; mingyangzhang@suda.edu.cn

Received 20 July 2020; Revised 27 November 2020; Accepted 5 December 2020; Published 23 December 2020

Academic Editor: Shi Yuan Xu

Copyright (C) 2020 Jiaxin Zhang et al. This is an open access article distributed under the Creative Commons Attribution License, which permits unrestricted use, distribution, and reproduction in any medium, provided the original work is properly cited.

\begin{abstract}
Ever since endogenous hydrogen sulfide $\left(\mathrm{H}_{2} \mathrm{~S}\right)$ was found in mammals in 1989, accumulated evidence has demonstrated that $\mathrm{H}_{2} \mathrm{~S}$ functions as a novel neurological gasotransmitter in brain tissues and may play a key role in traumatic brain injury. It has been proved that $\mathrm{H}_{2} \mathrm{~S}$ has an antioxidant, anti-inflammatory, and antiapoptosis function in the neuron system and functions as a neuroprotective factor against secondary brain injury. In addition, $\mathrm{H}_{2} \mathrm{~S}$ has other biologic effects such as regulating the intracellular concentration of $\mathrm{Ca}^{2+}$, facilitating hippocampal long-term potentiation (LTP), and activating ATP-sensitive $\mathrm{K}$ channels. Due to the toxic nature of $\mathrm{H}_{2} \mathrm{~S}$ when exceeding the physiological dose in the human body, only a small amount of $\mathrm{H}_{2} \mathrm{~S}$-related therapies was applied to clinical treatment. Therefore, it has huge therapeutic potential and has great hope for recovering patients with traumatic brain injury.
\end{abstract}

\section{Introduction}

Traumatic brain injury (TBI) is the leading cause of high mortality and high morbidity in young adults and a major cause of death and disability across all ages in all countries, with a huge burden on individuals and society in economic and healthy development. Significant progress has been made in providing treatment for TBI and achieving more equitable and sustained improvements across health services. Our group demonstrated for the first time that decreased endogenous hydrogen sulfide $\left(\mathrm{H}_{2} \mathrm{~S}\right)$ levels and $\mathrm{H}_{2} \mathrm{~S}$ synthesis enzyme cystathionine beta-synthase (CBS) expression in the brain were associated with increased lesion volume and mortality after TBI and the protective effect of exogenous $\mathrm{H}_{2} \mathrm{~S}$ on TBI [1-3]. Accumulating evidence has demonstrated the protective effect of $\mathrm{H}_{2} \mathrm{~S}$ on TBI $[1,2,4-8]$.

$\mathrm{H}_{2} \mathrm{~S}$ that smells disgusting odor is classified as the third gaseous transmitter following carbon monoxide and nitric oxide. Nowadays, evidence has demonstrated that $\mathrm{H}_{2} \mathrm{~S}$ was found to be produced endogenously in various parts of the body such as the heart, blood, and central nervous system (CNS) $[9,10]$. Moreover, it also refers to the actions of $\mathrm{H}_{2} \mathrm{~S}$ which is involved in the regulation of intracellular signaling molecules, ion channel function, and the release and function of amino acid neurotransmitters [11]. However, it was recently proposed that the neurological actions of $\mathrm{H}_{2} \mathrm{~S}$ were continuously modulated primarily by circulating sulfide rather than by endogenous production [12]. This was disproved based on recent studies showing that $\mathrm{H}_{2} \mathrm{~S}$ found in the CNS is more likely to be derived directly from the brain than from the blood [13, 14]. Moreover, investigations over the past two decades have shown that the concentration of $\mathrm{H}_{2} \mathrm{~S}$ has a detectable change going up or down which is related to brain injury. These results have shown that brainderived $\mathrm{H}_{2} \mathrm{~S}$ seems to serve as an important regulatory mechanism in the growth and development of neurons and the protection of neurons against brain injury.

In TBI, primary damage occurs at the time of impact and the damage is preventable but not treatable. The process will continue to cause following mitochondrial dysfunction, immune responses, the release of excitatory neurotransmitters, cerebrovascular dysfunction, and others that constitute the secondary injury [15]. In order to verify whether $\mathrm{H}_{2} \mathrm{~S}$ has the neuroprotective effect after injury, exogenous $\mathrm{H}_{2} \mathrm{~S}$ 
as an endogenous donor has been added into preclinical trials. Most of the neuroprotective drugs tested in mice have failed in human clinical trials because they target a single factor, which mediates secondary injury in TBI. Whether exogenous $\mathrm{H}_{2} \mathrm{~S}$ can affect brain-derived $\mathrm{H}_{2} \mathrm{~S}$ and has multiple neuroprotective effects on the secondary injury after TBI, it needs more evidence to verify. Therefore, this article focuses on the effective molecular mechanism of $\mathrm{H}_{2} \mathrm{~S}$ in TBI and puts forward some views on future research.

\section{The Severity of TBI}

In the clinical setting, the Glasgow Coma Scale (GCS) is a 15point behavioral observation scale that defines severity based on eye, verbal, and motor response. On the basis of direct observation of a limited number of objective variables, the GCS sums three scores to produce total scores of 3-8 (severe TBI), 9-12 (moderate TBI), and 13-15 (mild TBI). This scoring standard is also not absolute for dividing the severity of TBI. Moreover, there are still some limitations in judging some situations such as patients who are intubated and sedated or paralyzed and a wide range of injury magnitude represented by GCS scores of 3-8 [16]. Patients with GCS scores of 13 were considered moderate TBI for purposes in some studies. Moreover, computed tomography (CT) was used to assess the severity of TBI and a combination of clinical and imaging variables demonstrated a strong correlation with outcome when evaluated using the databases for several clinical trials. Subsequently, Magnetic Resonance Imaging (MRI) was also used as imaging evidence to participate in the assessment of damage. The Centers for Disease Control and Prevention provides one or more of the conditions about changes in consciousness and memory to define the severity of TBI. Therefore, other aforementioned clinical practice guidelines also incorporate structural imaging, duration of loss of consciousness and posttraumatic amnesia, and the GCS in their criteria for classifying injury severity (Table 1).

The establishment of the animal model is an excellent platform to delineate key injury mechanisms that associate with types of injury (concussion, contusion, and penetration injuries) that occur clinically for the investigation of mild, moderate, and severe forms of TBI [17], considering that the evaluation criteria for clinical use cannot be applied to the preclinical models. Therefore, well-defined grading guidelines for defining mild, moderate, and severe TBI in the rodent model are needed. Tissue loss about lesion size analysis is also recognized as a parameter to grade TBI severity. A number of studies utilized loss of cortical and hippocampal tissue to define TBI severity. Mild TBI has been defined as lesions confined to the cortical layer, a moderate injury was associated with considerable cortical tissue loss with little to no overt hippocampal loss, and severe TBI was often defined as extensive overt hippocampal lesions along with cortical tissue loss $[18,19]$. There are several common methods for establishing rodent TBI models, including the fluid percussion injury, controlled cortical impact (CCI), and weight-drop model [15]. CCI is well regarded because it allows researchers to quantify the relationship between measurable engineered parameters (e.g., force, velocity, and
TABLE 1: Characteristics of different degrees of TBI in different species.

\begin{tabular}{l}
\hline Species \\
\hline Mild TBI \\
(1) Any period of loss of consciousness up to 30 min \\
(2) Posttraumatic amnesia not exceeding $24 \mathrm{~h}$ \\
(3) Any period of confusion or disorientation \\
(4) Transient neurological abnormalities \\
(5) A GCS score of $13-15$ \\
(6) Normal structural imaging \\
(7) Postconcussion symptoms may resolve during \\
12 weeks \\
Moderate TBI \\
(1) A possible loss of consciousness lasting up to a \\
few hours \\
(2) Confusion lasting from days to weeks \\
(3) Physical, cognitive, and/or behavioral impairments \\
lasting for months \\
(1) Abnormal structural imaging \\
(2) A GCS score of 9-12 \\
Severe TBI \\
(1) Sustained loss of consciousness (>24h) \\
(2) Surviving patients exhibiting chronic physical and \\
emotional disabilities \\
(3) Abnormal structural imaging \\
(4) A GCS score of less than 9
\end{tabular}

\section{Mild TBI}

(1) CCI: depth: 0.1-1.0 mm; velocity: $3.0-6.0 \mathrm{~m} / \mathrm{s}$

(2) Tissue loss: lesions confined to the cortical layer

(3) Cortical depression $<0.5 \mathrm{~mm}$, velocities $<4.0 \mathrm{~m} / \mathrm{s}$

Moderate TBI

(1) CCI: depth: $0.5-3.0 \mathrm{~mm}$; velocity: $1.5-6.0 \mathrm{~m} / \mathrm{s}$

(2) Tissue loss: considerable cortical tissue loss with little

Mouse to no overt hippocampal loss

(3) Cortical depression $1.0-1.5 \mathrm{~mm}$, velocities $4.0-5.0 \mathrm{~m} / \mathrm{s}$

Severe TBI

(1) CCI: depth: $0.5-2.0 \mathrm{~mm}$; velocity: $3.0-6.0 \mathrm{~m} / \mathrm{s}$

(2) Tissue loss: extensive overt hippocampal lesions along with cortical tissue loss

(3) Cortical depression $>2.0 \mathrm{~mm}$, velocities $>5.0 \mathrm{~m} / \mathrm{s}$

Note: TBI: traumatic brain injury; CCI: controlled cortical impact; GCS: the Glasgow Coma Scale.

depth of tissue deformation) and the extent of (either functional and/or tissue) impairment [20]. The CCI model was originally developed to investigate moderate to severe TBI and is infrequently used to mimic mild TBI because of the necessity of a craniectomy. However, modified versions of CCI have now been developed which means that CCI can induce the TBI model with different degrees of injury. According to previous articles, for mild TBI, the depth of impact in the cortex ranged from 0.1 to $1.0 \mathrm{~mm}$ and velocity ranged from 3.0 to $6.0 \mathrm{~m} / \mathrm{s}$; for moderate TBI, the depth of impact in the cortex ranged from 0.5 to $3.0 \mathrm{~mm}$ and velocities of $1.5-6.0 \mathrm{~m} / \mathrm{s}$, and severe injury used impact depths of $0.5-2.0 \mathrm{~mm}$ and velocities of $3.0-6.0 \mathrm{~m} / \mathrm{s}$ [15, 20-23]. Many studies use a composite neurological evaluation to assess the severity of motor deficits following TBI, but the 
problem is still that the assessment criteria cannot be unified. Thus, CCI affords pristine ability to analyze the biomechanical parameters of injury of interest in TBI research.

Although each case of TBI is unique and affected individuals display different degrees of injury (Table 1), we will discuss some of the common underlying neurochemical and molecular mechanisms and common secondary events following different severity of TBI.

\section{Production and Storage of Endogenous $\mathrm{H}_{2} \mathrm{~S}$}

Under physiological conditions, endogenous $\mathrm{H}_{2} \mathrm{~S}$ in human bodies is generated via enzymatic and nonenzymatic pathways [24]. $\mathrm{H}_{2} \mathrm{~S}$ via nonenzymatic pathways is mainly produced by the decomposition of an inorganic substance, which accounts for a little percentage of $\mathrm{H}_{2} \mathrm{~S}$ production. The main generation of $\mathrm{H}_{2} \mathrm{~S}$ in the human body mainly depends on the enzymatic pathways using L-cysteine as the substrate. Three types of enzymes play a key role in these pathways, including cystathionine- $\beta$-synthase (CBS), cystathionine- $\gamma$-lyase (CSE), and 3-mercaptopyruvate transferase (3-MST) [25]. CBS hydrolyzes cysteine to produce $\mathrm{H}_{2} \mathrm{~S}$, with $\mathrm{L}$-serine as the by-product. Furthermore, CBS could catalyze the condensation of cysteine and homocysteine to form cystathionine and $\mathrm{H}_{2} \mathrm{~S}$. The release of $\mathrm{H}_{2} \mathrm{~S}$ could also depend on the reaction on the thiol of cysteine by CBS catalyzing to form s-thiolate. In addition, cysteine also may be hydrolyzed by CSE to produce $\mathrm{H}_{2} \mathrm{~S}$ with the concomitant production of pyruvate and ammonia. In addition to CBS and CSE, 3MST as a pyridoxal-5'-phosphate-independent enzyme is also involved in the production of $\mathrm{H}_{2} \mathrm{~S}$ and is identified in the neurons. 3-MST acts together with cysteine aminotransferase (CAT) to generate $\mathrm{H}_{2} \mathrm{~S}$ from cysteine (Cys) in the presence of $\alpha$-ketoglutarate. The additional pathway for $\mathrm{H}_{2} \mathrm{~S}$ biosynthesis has been reported that 3-MST along with D-amino acid oxidase (DAO) produces $\mathrm{H}_{2} \mathrm{~S}$ from $\mathrm{D}$ cysteine by the interaction of mitochondria and peroxisomes which occurs mainly in the cerebellum and the kidney. Both CBS and CSE are pyridoxal- $5^{\prime}$-phosphate-dependent enzymes, but they mediate, respectively, the production of $\mathrm{H}_{2} \mathrm{~S}$ in different tissues and organs. CBS is expressed mainly in the central nervous system, liver, and kidney while CSE is expressed mainly in the vascular smooth muscle, nonvascular smooth muscle, and a little in the liver, kidney, uterus, placenta, pancreas, and other organs [26]. The location of these enzymes in different tissues is very important because the regulation of endogenous $\mathrm{H}_{2} \mathrm{~S}$ production can be achieved by targeting each enzyme separately or simultaneously.

Under physiological conditions $(\mathrm{pH}=7.4), \mathrm{H}_{2} \mathrm{~S}$ largely exists in two forms: the neutral molecular form $\left(\mathrm{H}_{2} \mathrm{~S}\right)$ and an ionic form $\left(\mathrm{HS}^{-}\right)$. The two forms are able to transform into each other and maintain the dynamic equilibrium at a ratio of one to two [27]. Although endogenous $\mathrm{H}_{2} \mathrm{~S}$ can be synthesized and released immediately, the storage forms of $\mathrm{H}_{2} \mathrm{~S}$ are also known. The acid-labile pool and the sulfane sulfur pool, which include hydrodisulfides/persulfides, are accepted storage forms of $\mathrm{H}_{2} \mathrm{~S}$ ([28]), of which the acid-labile pool consists of iron-sulfur-containing proteins located in mitochondrial enzymes and can only release $\mathrm{H}_{2} \mathrm{~S}$ at an acid $\mathrm{pH}$ of 5.4. The sulfane sulfur pool is localized in the cytoplasm and releases $\mathrm{H}_{2} \mathrm{~S}$ under reducing conditions of $\mathrm{pH}$ 8.4 [29]. Because mitochondria are not in the acidic condition, acid-labile sulfur may not be a physiologic source of $\mathrm{H}_{2} \mathrm{~S}$. Free $\mathrm{H}_{2} \mathrm{~S}$ is immediately absorbed and stored as bound sulfur. 3-MST and CAT are located in the mitochondria and can produce $\mathrm{H}_{2} \mathrm{~S}$ from cysteine in the brain [30]. It is speculated that $\mathrm{H}_{2} \mathrm{~S}$ produced by the 3-MST/CAT enzymatic pathway is stored in the bound sulfane sulfur pool $[9,31]$.

\section{The Change of Endogenous Hydrogen Sulfide in TBI}

Up to now, multiple experiments have been designed to examine and describe the change of $\mathrm{H}_{2} \mathrm{~S}$ concentration after TBI both in animal models and in human patients. Our group demonstrated for the first time that the concentration of $\mathrm{H}_{2} \mathrm{~S}$ presented a dynamic change in the TBI model. The level of endogenous $\mathrm{H}_{2} \mathrm{~S}$ and CBS expression in the blood and brain exhibits a downtrend after TBI $[2,3]$. Recently, our group also reported that pretreated with $\mathrm{NaHS}, \mathrm{a}_{2} \mathrm{~S}$ donor, a limited lesion volume in the ipsilateral cortex has been observed in the TBI model of mice [1]. Jiang et al. reported $\mathrm{NaHS}$ treatment increased the $\mathrm{H}_{2} \mathrm{~S}$ level of brain tissue and endogenous antioxidant enzymatic activities and decreased oxidative product levels in the brain tissue of TBI-challenged rats [5]. Campolo et al. reported that ATB346 , a hydrogen sulfide-releasing derivative of naproxen, significantly reduced the severity of inflammation and restored neurotrophic factors that characterized the secondary events of TBI [4]. Karimi et al. reported that NaHS has a neuroprotective effect on TBI-induced memory impairment in rats [6]. $\mathrm{Xu}$ et al. reported that NaHS restores mitochondrial function and inhibits autophagy by activating the PI3K/Akt/mTOR signaling pathway to improve functional recovery after TBI [7]. The experimental evidence indicates that $\mathrm{H}_{2} \mathrm{~S}$ functions as an important neuroprotective mediator in TBI models and may offer clinical therapy for TBI treatment.

\section{Biologic Effect of Exogenous Hydrogen Sulfide in TBI}

Ever since $\mathrm{H}_{2} \mathrm{~S}$ was described for the first time in 1713, the toxicity of $\mathrm{H}_{2} \mathrm{~S}$ to the human has become the focus of everyone's attention. $\mathrm{H}_{2} \mathrm{~S}$ is often considered as one of the most unusual and reliable toxic gas [32]. $\mathrm{H}_{2} \mathrm{~S}$ exerts its toxic effects characterized by acute central neurotoxicity, pulmonary edema, conjunctivitis, and odor perception followed by respiratory paralysis which is mainly due to the binding of sulfide to cytochrome c oxidase in mitochondria. Clinical symptoms of acute sulfide poisoning are featured by memory loss and brain dysfunction, which can be explained by the process that excess $\mathrm{H}_{2} \mathrm{~S}$ in brain tissue lowers the level of neurotransmitters by inhibiting monoamine oxidase [10]. However, in recent years with the increasing study on $\mathrm{H}_{2} \mathrm{~S}$, its biological effect is gradually being recognized, especially its neuroprotective ability after TBI (Figure 1). 


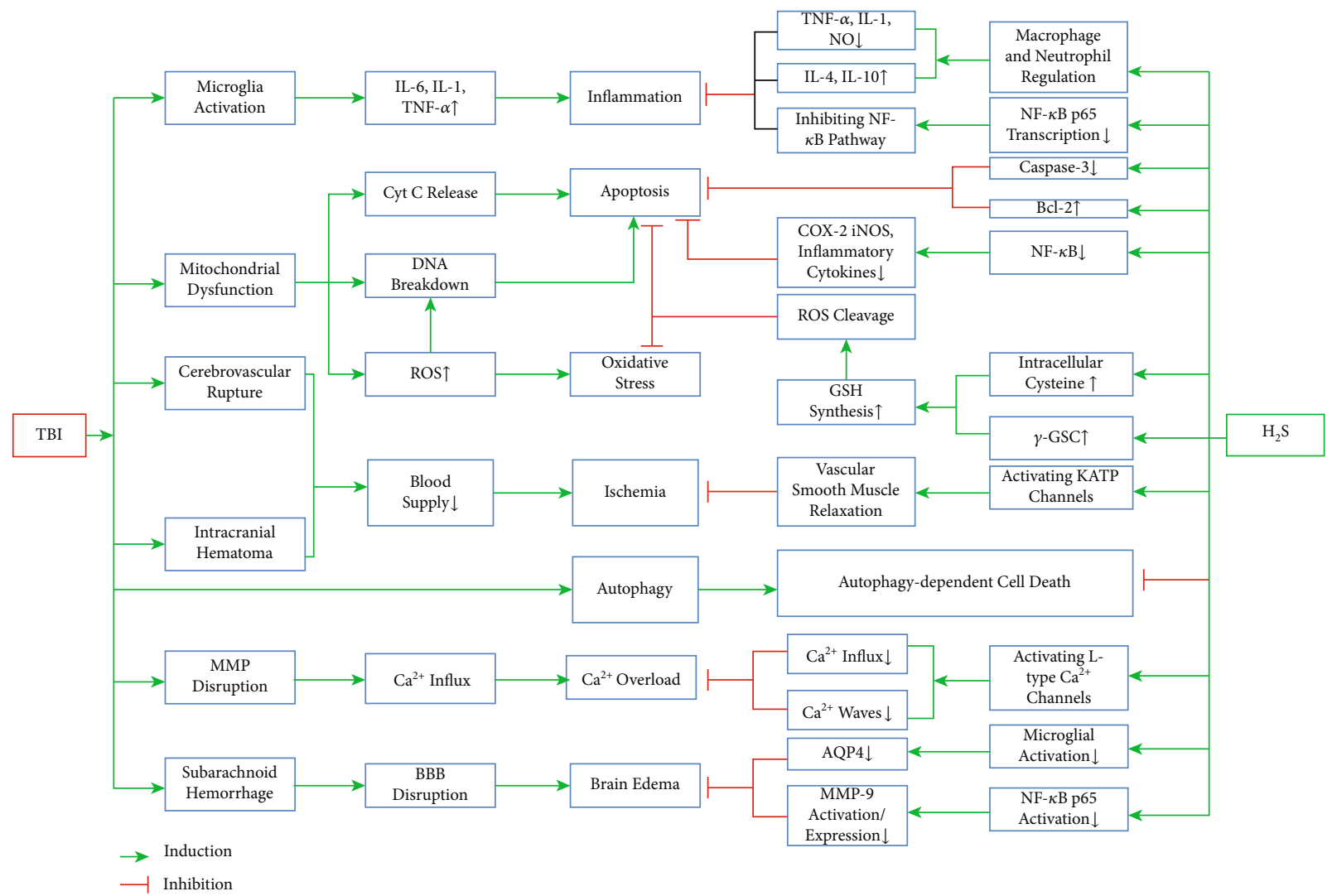

Figure 1: The potential therapeutic effects of $\mathrm{H}_{2} \mathrm{~S}$ on TBI. The diagram shows the mechanisms of TBI-induced pathological change in the brain tissues and the nervous system, along with part of the neuroprotective pathways of $\mathrm{H}_{2} \mathrm{~S}$. Note: $\uparrow$ : upregulated; $\downarrow$ : downregulated.

5.1. Anti-Inflammatory Role of $\mathrm{H}_{2} \mathrm{~S}$. Neuroinflammation, which is characterized by the activation of glial cells, recruitment of neutrophils and macrophages, and upregulation of cytokines, adhesion factors, and chemokines, can be triggered from the surrounding to the site of injury and cause secondary neuronal injury after TBI $[33,34]$. The pioneering work revealed the anti-inflammatory properties of $\mathrm{H}_{2} \mathrm{~S}$ in different disease models. In the TBI model, the neuroprotective effect of $\mathrm{H}_{2} \mathrm{~S}$ in inflammatory is generally achieved by the regulation of macrophages and neutrophils. $\mathrm{H}_{2} \mathrm{~S}$ inhibits the release of proinflammatory factors such as tumor necrosis factor- $\alpha$ (TNF- $\alpha)$, interleukin- $1 \beta$ (IL- $1 \beta)$, and NO from astrocytes and microglial cells [10] and increases anti-inflammatory cytokines like interleukin-4 (IL-4) or interleukin-10 (IL-10) [35]. One of the possible antiinflammatory mechanisms is that $\mathrm{H}_{2} \mathrm{~S}$ inhibits the iNOS, NF- $\kappa$ B, ERK, and p38 MAPK signaling pathways [36]. $\mathrm{NF}-\kappa \mathrm{B}$ transcription factors are ubiquitously expressed in mammalian cells and are known to upregulate the expressions of cytokines and chemokines. The NF- $\kappa$ B family (also known as the Rel family) consists of five members: p50, p52, p65 (also known as Rel A), c-Rel, and Rel B. Inflammation begins to be mediated when activated NF- $\kappa$ B enters the nucleus to induce transcription of a myriad of genes. It has been reported that ATB-346, a hydrogen sulfide-releasing cyclooxygenase inhibitor, significantly reduced the translocation of the p65 submit following TBI in rats [4]. Xiang et al. showed that $\mathrm{H}_{2} \mathrm{~S}$ alleviates lipopolysaccharide- (LPS-) induced inflammatory via inhibition of NF- $\kappa \mathrm{B}$ p65 translocation [37]. In addition, $\mathrm{H}_{2} \mathrm{~S}$ can also polarize microglia to an anti-inflammatory phenotype (M2) by activating calmodulindependent protein kinase $\beta$ - $(\mathrm{CaMKK} \beta$ - $)$ dependent AMPactivated protein kinase (AMPK) [38]. However, the role of $\mathrm{H}_{2} \mathrm{~S}$ in the inflammatory reaction still remains controversial. Some groups have observed that $\mathrm{H}_{2} \mathrm{~S}$ plays a proinflammatory role in severe burn injury and LPS-induced inflammation $[39,40]$. Consequently, $\mathrm{H}_{2} \mathrm{~S}$ is currently accepted to be one of the pivotal factors that regulate the inflammatory reaction following $\mathrm{TBI}$, but the precise mechanism that $\mathrm{H}_{2} \mathrm{~S}$ modulates inflammation remains to be elucidated.

5.2. Antioxidant Role of $\mathrm{H}_{2} \mathrm{~S}$. During the process of TBI, the physical and secondary damage at the trauma site result in mitochondrial dysfunction and changes in membrane permeability, causing the breakdown of the balance between the generation and elimination of intracellular reactive oxygen species (ROS) (Campolo et al.). Then, tremendous intracellular accumulation of ROS induces cytotoxicity, causing a large amount of cell death.

Oxygen consumption of brain tissue accounts for nearly $20 \%$ of the entire body, making brain tissue more sensitive to oxidative stress-related disorders such as TBI, stroke, and Alzheimer's disease [40, 41]. Glutathione (GSH), a tripeptide consisting of cysteine, glutamate, and glycine, is a major antioxidant in the cellular defense against oxidative stress, and $\mathrm{H}_{2} \mathrm{~S}$ can promote the production of GSH ([42]; Kimura and 
[43]). The intracellular concentration of cysteine is much lower than the other two substrates of GSH [44], and cysteine exists in the extracellular space in an oxidized form, cystine [45], so the availability of intracellular cysteine significantly determined the rate of GSH synthesis. Evidence has shown that $\mathrm{H}_{2} \mathrm{~S}$ reduces cysteine into cystine and activates the cysteine/glutamate antiporter (xc-) which couples the import of cysteine and export of glutamate [46], consequently increasing the intracellular concentration of cysteine. On the other hand, $\mathrm{H}_{2} \mathrm{~S}$ is able to potentiate the activity of $\gamma$-glutamylcysteine synthase ( $\gamma$-GSC), which functions as a ratelimiting enzyme in the production of GSH (Kimura and [43]). In addition, $\mathrm{H}_{2} \mathrm{~S}$ may have a synergistic effect with other antioxidative systems including haem oxygenase (HO), superoxide dismutase (SOD), and especially nuclear factor erythroid-2-related factor2 (Nrf2) [47]. More evidence shows $\mathrm{H}_{2} \mathrm{~S}$ can induce the upregulation of $\mathrm{Nrf2}$ and drive it into the nucleus, and it can regulate the level of antioxidant enzymes and enhance the antioxidant reaction which is certified in the diverse damage models [48]. Meanwhile, it is reported that $\mathrm{H}_{2} \mathrm{~S}$ can reduce the production of prooxidase, which still represents the inhibition of the occurrence of oxidative stress [49]. It is worth pointing out that $\mathrm{H}_{2} \mathrm{~S}$ produced by 3-MST, which is localized in the mitochondria, can directly reduce the generation of ROS and protects cells [50]. Our group reported that $\mathrm{H}_{2} \mathrm{~S}$ protects against cell damage induced by scratch injury through modulation of the PI3K/Akt/Nrf2 pathway, suggesting $\mathrm{H}_{2} \mathrm{~S}$ may have therapeutic efficacy in TBI [51].

5.3. Regulation of Cell Death Signaling Mediated by $\mathrm{H}_{2} \mathrm{~S}$. Neuronal cell death following TBI is an important factor in neurological deficits. TBI can trigger localized neuronal apoptosis within a few hours after the trauma. Increased cytosolic free $\mathrm{Ca}^{2+}$ can induce the collapse of the mitochondrial membrane potential and the production of free radicals and lipid peroxidation. This leads to further attack on the mitochondrial membranes, which leads to the release of cytochrome $\mathrm{C}$ through the outer mitochondrial membrane. Then, cytochrome $\mathrm{C}$ enters the cytoplasm, inducing caspase-dependent apoptosis [52]. Meanwhile, ROS produced from dysfunctional mitochondria can also induce neuronal apoptosis by directly causing the breakdown of DNA. As is discussed above, part of the antiapoptotic effect of $\mathrm{H}_{2} \mathrm{~S}$ is achieved through the inhibition of the ROS injury. It has been observed that $\mathrm{H}_{2} \mathrm{~S}$ is able to inhibit the $\mathrm{H}_{2} \mathrm{O}_{2}$ activated calcium signaling pathways in mouse hippocampal neurons [53]. In the model of oxygen-glucose deprivation/ reoxygenation- (OGD/R-) induced neuronal apoptosis, $\mathrm{H}_{2} \mathrm{~S}$ is observed to inhibit a ROS-mediated caspase-3 pathway [54]. Ji et al. found that $\mathrm{H}_{2} \mathrm{~S}$ preconditioning considerably reduced TUNEL-positive cells and cleaved caspase- 3 in the cerebral ischemia/reperfusion injury model, and the protective effects of $\mathrm{H}_{2} \mathrm{~S}$ are possibly achieved by the induction of HSP70 through the PI3K/Akt/Nrf2 pathway [55]. Caspase3 , Bcl-2, and Bax protein function as important mediators in the TBI-induced apoptosis [56]. We found that NaHS pretreatment can reverse the effects on the cleaved caspase- 3 increase and Bcl-2 decrease in the injured cortex and hippo- campus after TBI [1]. In addition, $\mathrm{H}_{2} \mathrm{~S}$ plays another antiapoptotic role via the regulation of the NF- $\kappa \mathrm{B}$ signaling pathway, which plays a complex role in integrating signaling between and within neurons and glial. $\mathrm{H}_{2} \mathrm{~S}$ reduces the expression of NF- $\kappa \mathrm{B}$ followed by the decrease of the expression of iNOS, COX-2, and proinflammatory cytokines. Sen et al. showed that $\mathrm{H}_{2} \mathrm{~S}$ sulfhydrate, the p65 subunit of NF- $\kappa \mathrm{B}$ at cysteine- 38 which augments its ability to bind its coactivator RPS3 and the activator/coactivator complex, then stimulates the transcription of antiapoptotic genes [57].

Autophagy is a physiological process that helps maintain a balance between the manufacture of cellular components and breakdown of damaged organelles and other toxic cellular constituents [58]. Sarkar et al. have shown that autophagic clearance is impaired early after TBI and correlates with neuronal cell death [59]. The Nomenclature Committee of Cell Death defines autophagy-dependent cell death as "a form of regulated cell death that mechanistically depends on the autophagic machinery" and "a distinct mechanism of cell death that occurs independently of apoptosis or necrosis" $[60,61]$. Autophagy as an attractive therapeutic target can be developed new therapeutic strategies to achieve better outcomes for patients suffering from TBI [62]. Our group has shown that $\mathrm{H}_{2} \mathrm{~S}$ regulated autophagy-dependent cell death after TBI $[1,51,63]$. We also showed that 3-MST was mainly located in living neurons and may be implicated in the autophagy of neurons and involved in the pathophysiology of the brain after TBI [8].

5.4. Cerebral Vasodilation Mediated by $\mathrm{H}_{2} \mathrm{~S}$. Research conducted in laboratory animals and humans has investigated the effects of TBI on cerebral blood flow (CBF). Many investigations have revealed that focal or global cerebral ischemia occurs frequently by detection within a temporal range from ultraearly to late stages after TBI. The mechanism may be that the rupture of cerebral vessels, nerve parenchyma, and edema formed by intracranial hematoma may block blood flows, resulting in insufficient blood supply. After TBI, CBF autoregulation, which refers to cerebrovascular constriction or dilation in response to increases or decreases in cerebral perfusion pressure (CPP), is impaired or abolished in most patients. The defect of automatic adjustment of cerebral blood flow occurs in an uncertain time after injury, which can occur immediately after the trauma or may develop at other times after injury. Furthermore, about more than one-third of TBI patients show cerebral vasospasm, and in all patients with vasospasm, 50\% have hypoperfusion. In this process, the destruction of neuronal membrane and dysfunction of various ion channels located on the membrane can cause disrupted ion homeostasis, during which $\mathrm{Na}^{+}$and $\mathrm{Ca}^{2+}$ accumulate in the cytoplasm and a large amount of $\mathrm{K}^{+}$is pumped out. As a result, the brain cells become sensitive to ischemia [64]. Multiple studies have proved that $\mathrm{H}_{2} \mathrm{~S}$ dilates cerebral vessels following TBI via activation of the ATP-sensitive $\mathrm{K}$ channels ( $\mathrm{K}_{\mathrm{ATP}}$ channels) [5]. According to reports, specific molecular targets of $\mathrm{H}_{2} \mathrm{~S}$ appear to be cysteine 6 and 26 in the extracellular portion of the rvSUR1 subunit of the $\mathrm{K}_{\mathrm{ATP}}$ channel complex. The opening of the $\mathrm{K}_{\mathrm{ATP}}$ channels inactivates cell membrane polarization and voltage- 
TABLE 2: Summary of the biologic effects of hydrogen sulfide after TBI.

\begin{tabular}{|c|c|c|}
\hline Effects & Mechanisms & References \\
\hline \multirow[t]{3}{*}{ Anti-inflammation } & Inhibiting the TNF- $\alpha$, IL- $1 \beta$, and NO & {$[10]$} \\
\hline & Increasing the IL-6 and IL-10 & [35] \\
\hline & Inhibiting the iNOS, NF- $\kappa \mathrm{B}, \mathrm{ERK}$, and p38 MAPK pathways & {$[36]$} \\
\hline \multirow[t]{4}{*}{ Antioxidation } & Activation of cysteine/glutamate antiporter & {$[46]$} \\
\hline & Activation of $\gamma$-GCS & (Kimura and [43]) \\
\hline & Cooperating with $\mathrm{HO}$, SOD, and $\mathrm{Nrf} 2$ antioxidative system & (Kimura and [43]) \\
\hline & Decreasing the production of the prooxidase & (Kimura and [43]) \\
\hline \multirow[t]{3}{*}{ Antiapoptosis } & Inhibiting the $\mathrm{H}_{2} \mathrm{O}_{2}$-activated calcium pathways & {$[53]$} \\
\hline & Reducing caspase- 3 and increasing Bcl-2 & [54] \\
\hline & Regulating the NF- $\kappa \mathrm{B}$ signaling pathway & {$[57]$} \\
\hline Regulating autophagy-dependent cell death & $\begin{array}{c}\text { Reducing Beclin-1 and LC-3 } \\
\text { LC3-positive cells were partly colocalized with PI }\end{array}$ & $\begin{array}{c}{[1]} \\
{[8]} \\
{[63]} \\
{[51]}\end{array}$ \\
\hline Vasodilation & $\begin{array}{l}\text { Activating the } \mathrm{K}_{\text {ATP }} \text { channels (CSE-generated } \mathrm{H}_{2} \mathrm{~S} \text { ) } \\
\text { Liberating NO from S-nitrosothiols }\end{array}$ & $\begin{array}{l}{[65]} \\
{[78]}\end{array}$ \\
\hline \multirow[t]{2}{*}{$\mathrm{Ca}^{2+}$ modulation } & Activating the L-type $\mathrm{Ca}^{2+}$ channels & {$[68]$} \\
\hline & Inducing $\mathrm{Ca}^{2+}$ waves & {$[70]$} \\
\hline Attenuating edema & Alleviating BBB disruption and reducing AQP4 expression & {$[72]$} \\
\hline Facilitating LTP & Potentiating the NMDA receptor & {$[77]$} \\
\hline Antiexcitotoxicity & Reducing glutamate release after TBI & {$[64]$} \\
\hline
\end{tabular}

gated calcium channels, which in turn lead to a reduction in $\mathrm{Ca}^{2+}$, which ultimately leads to the relaxation and expansion of blood vessels. In a vitro model of rat aortic tissues, $\mathrm{H}_{2} \mathrm{~S}$ activates $\mathrm{K}_{\mathrm{ATP}}$ channels by inducing the efflux of intracellular potassium and the hyperpolarization of the membrane of vascular smooth muscle cells [65]. Later, another group reported that $\mathrm{H}_{2} \mathrm{~S}$ has a similar mechanism of vasodilation in the cerebral cortical pial arteriole of newborn pigs [66]. However, conflicting reports have emerged showing that the contribution of the $\mathrm{K}_{\text {ATP }}$ channels to $\mathrm{H}_{2} \mathrm{~S}$-induced vasodilation is minimal and that vasodilation is due to metabolic inhibition such as the decrease in ATP, intracellular $\mathrm{pH}$ changes, and modulation of $\mathrm{Cl}^{-} / \mathrm{HCO}_{3}{ }^{-}$channels. There are also indications that $\mathrm{H}_{2} \mathrm{~S}$ may stimulate vasodilation by liberating NO from S-nitrosothiols. Although the mechanism of cerebral vasodilation is still unclear, it is certain that hydrogen sulfide has the effect of dilating cerebral blood vessels after TBI.

5.5. $\mathrm{H}_{2} \mathrm{~S}$ Mediates Intracellular Calcium Concentration. After the primary injury of TBI, the disruption of mitochondrial membrane potential (MMP) and intracellular excessive ROS lead to intracellular calcium accumulation, causing calcium overload-induced neurotoxicity [67]. $\mathrm{Ca}^{2+}$ activates lipid peroxidases, proteases, and phospholipases which in turn increase the intracellular concentration of free fatty acids and free radicals. Calcium overload, which is at the core of the cellular and molecular network of secondary neuronal injury, is involved in three neurotoxic cascade reactions leading to neuronal apoptosis or necrosis [64]. It has been noted that $\mathrm{H}_{2} \mathrm{~S}$ is capable of regulating $\mathrm{Ca}^{2+}$ in all important brain cell types, namely, neurons, microglia, and astrocytes. The physiological concentrations of $\mathrm{H}_{2} \mathrm{~S}$ selectively were initially found to be a neuromodulator and facilitate the induction of hippocampal long-term potentiation (LTP) by enhancing the activity of $\mathrm{N}$-methyl-D-aspartate (NMDA) receptors in neurons and increase the influx of $\mathrm{Ca}^{2+}$ into astrocytes. $\mathrm{H}_{2} \mathrm{~S}$ could raise cytosolic calcium in neurons through the activation of L-type $\mathrm{Ca}^{2+}$ channels. Some groups have observed a concentration-dependent $\mathrm{H}_{2} \mathrm{~S}$-induced $\mathrm{Ca}^{2+}$ elevation in neurons, and such effects of $\mathrm{H}_{2} \mathrm{~S}$ on cytosolic $\mathrm{Ca}^{2+}$ concentration can be attenuated by the antagonist of L-type calcium channel and N-methyl-D-aspartate receptor $[68,69]$. In TBI, $\mathrm{H}_{2} \mathrm{~S}$ released from neurons or glia in response to neuronal excitation activates $\mathrm{Ca}^{2+}$ channels of astrocytes, inducing $\mathrm{Ca}^{2+}$ waves that propagate to the neighboring astrocytes [70], and $\mathrm{H}_{2} \mathrm{~S}$ may mediate signals between neurons and glia. However, there are still some reports suggesting that $\mathrm{H}_{2} \mathrm{~S}$ elevates neuronal $\mathrm{Ca}^{2+}$ concentration and may exacerbate the formation of calcium overload in secondary neuronal injury [64].

5.6. $\mathrm{H}_{2} \mathrm{~S}$ Attenuates the TBI-Induced Brain Edema. Brain edema, which is usually formed in the early brain injury following subarachnoid hemorrhage, can result in brain swelling and increased intracranial pressure followed with neuronal cell death, herniation, and death [71]. Our group 
reported that TBI significantly increases the percentage of water content in the injured ipsilateral cortex, leading to the formation of TBI-induced brain edema [1]. It is also reported that exogenous $\mathrm{H}_{2} \mathrm{~S}$ treatment can effectively ameliorate the development of TBI-induced brain edema [1]. Another group demonstrated that the effect of $\mathrm{H}_{2} \mathrm{~S}$ on the inhibition of brain edema formation might be involved with alleviating blood-brain barrier (BBB) disruption and reducing aquaporin-4 (AQP4) expression, both of which are induced by matrix metalloprotease- (MMP-) mediated tight junction proteins (TJPs) [72]. Classically, brain edema was thought to be triggered by the disruption of $\mathrm{BBB}$ integrity in the acute stage of subarachnoid hemorrhage [73]. Then, the degeneration of the basal lamina and the increased permeability of $\mathrm{BBB}$ result in the extracellular accumulation of water in the cortex [74]. In recent years, however, multiple investigations demonstrated that the dysfunction of ion and water channels plays a vital role in glial and neuron swelling [75]. Evidence showed that the deletion of AQP4, a main water channel protein concentrated on the astrocyte endfeet, protects $\mathrm{BBB}$ integrity by reducing inflammatory responses due to the upregulation of PPAR- $\gamma$ expression and attenuation of proinflammatory cytokine release [76]. In addition, studies also proved that $\mathrm{H}_{2} \mathrm{~S}$ attenuates brain edema by inhibiting the expression/activation of MMP-9, which is possibly associated with the regulation of $\mathrm{NF}-\kappa \mathrm{B}$ p 65 activation [72]. It is now demonstrated that $\mathrm{H}_{2} \mathrm{~S}$ may attenuate the AQP4-induced cellular edema via inhibition of microglial activation and suppression of proinflammatory cytokine release in the cortex, but the underlying mechanisms of $\mathrm{H}_{2} \mathrm{~S}$ alleviating brain edema through cellular pathways still demand further investigation.

5.7. Other Mechanisms of $\mathrm{H}_{2} \mathrm{~S}$ following TBI. Apart from the biologic effects of $\mathrm{H}_{2} \mathrm{~S}$ following TBI mentioned above, the underlying mechanisms of $\mathrm{H}_{2} \mathrm{~S}$ are reflected in many other aspects. It was found that $\mathrm{H}_{2} \mathrm{~S}$ facilitates hippocampus long-term potentiation via mediation of the N-methyl-Daspartate receptor in the $\mathrm{H}_{2} \mathrm{~S}$-activated cAMP/PKA pathway $[11,77]$. Moreover, $\mathrm{H}_{2} \mathrm{~S}$ also counteracts glutamatemediated excitotoxicity in the secondary injury following TBI via activation of $\mathrm{K}_{\mathrm{ATP}}$ channels, which may directly result in neuronal glutamate release in response to calcium influx after TBI [64].

According to all that have been mentioned above, the mechanisms of $\mathrm{H}_{2} \mathrm{~S}$ following TBI can be summarized in Table 2.

\section{Conclusion}

Although $\mathrm{H}_{2} \mathrm{~S}$ has multiple biologic effects as a neuroprotective gasotransmitter and considerable part of them has already been confirmed through experiments in laboratory, applying $\mathrm{H}_{2} \mathrm{~S}$-related therapy to clinical treatment still has a long way to go. $\mathrm{H}_{2} \mathrm{~S}$ can function as a neuroprotective mediator when it is controlled within physiological dose, while excess $\mathrm{H}_{2} \mathrm{~S}$ has chemotoxic and cytotoxic effects on human bodies [79]. $\mathrm{H}_{2} \mathrm{~S}$ still remains many potential mechanisms which have not been totally figured out, but it is beyond doubt that $\mathrm{H}_{2} \mathrm{~S}$ therapy, especially on TBI patients, will become an available treatment option in the near future.

\section{Abbreviations}

3-MST: 3-Mercaptopyruvate transferase

$\gamma$-GCS: $\quad \gamma$-Glutamylcysteine synthase

AMPK: AMP-activated protein kinase

AQP4: Aquaporin-4

ATB-346: 2-(6-Methoxynapthalen-2-yl)-propionic acid 4-thiocarbamoyl-phenyl ester

ATP: $\quad$ Adenosine triphosphate

BBB: Blood-brain barrier

Bcl-2: B-cell lymphoma-2

CaMKK $\beta$ : Calmodulin-dependent protein kinase $\beta$

cAMP: Cyclic adenosine monophosphate

CAT: $\quad$ Cysteine aminotransferase

CBF: $\quad$ Cerebral blood flow

CBS: $\quad$ Cystathionine- $\beta$-synthase

CCI: $\quad$ Controlled cortical impact

CNS: Central nervous system

CPP: Cerebral perfusion pressure

CSE: $\quad$ Cystathionine- $\gamma$-lyase

CT: $\quad$ Computed tomography

Cys: $\quad$ Cysteine

DAO: D-Amino acid oxidase

ERK: $\quad$ Extracellular signal-regulated kinase

GCS: Glasgow Coma Scale

GSH: Glutathione

$\mathrm{H}_{2} \mathrm{O}_{2}$ : Hydrogen peroxide

$\mathrm{H}_{2} \mathrm{~S}: \quad$ Hydrogen sulfide

HO: Haem oxygenase

HSP70: $\quad$ Heat shock protein 70

IL-1 $\beta$ : Interleukin- $1 \beta$

IL-4: $\quad$ Interleukin-4

IL-10: Interleukin-10

INOS: Inducible nitric oxide synthase

LPS: Lipopolysaccharide

LTP: Long-term potentiation

MAPK: Mitogen-activated protein kinase

MMP: Mitochondrial membrane potential

MMPs: Matrix metalloproteases

MRI: $\quad$ Magnetic Resonance Imaging

mTOR: Mammalian/mechanistic target of rapamycin

NaHS: $\quad$ Sodium hydrosulfide hydrate

NF- $\kappa$ B: Nuclear factor-kappa B

NMDA: N-Methyl-D-aspartate

NO: $\quad$ Nitric oxide

Nrf2: $\quad$ Nuclear factor erythroid-2 related factor2

OGD/R: Oxygen-glucose deprivation/reoxygenation

PI: $\quad$ Propidium iodide

PI3K: $\quad$ Phosphoinositide 3-kinase

PKA: $\quad$ Protein kinase A

PPAR- $\gamma$ : Peroxisome proliferator-activated receptors $\gamma$

ROS: $\quad$ Reactive oxygen species

SOD: $\quad$ Superoxide dismutase

TBI: $\quad$ Traumatic brain injury 
TJPs: $\quad$ Tight junction proteins

TNF- $\alpha$ : Tumor necrosis factor- $\alpha$

TUNEL: Terminal deoxynucleotidyl transferase-mediated dUTP-biotin in situ nick-end labeling.

\section{Conflicts of Interest}

The authors declare that they have no conflict of interest.

\section{Authors' Contributions}

Jiaxin Zhang and Shaoyi Zhang contributed equally to this study.

\section{Acknowledgments}

This study was funded by the National Natural Science Foundation of China (No. 82071382 and No. 81601306), the Priority Academic Program Development of Jiangsu Higher Education Institutions (PAPD), the Jiangsu Talent Youth Medical Program (QNRC2016245), the Jiangsu Maternal and Child Health Research Key Project (F202013) and the Suzhou Science and Technology Development Project (SYS2020089 and SYS2018082).

\section{References}

[1] M. Zhang, H. Shan, P. Chang et al., "Hydrogen Sulfide Offers Neuroprotection on Traumatic Brain Injury in Parallel with Reduced Apoptosis and Autophagy in Mice," PLoS ONE, vol. 9, no. 1, p. e87241, 2014.

[2] M. Zhang, H. Shan, T. Wang et al., "Dynamic Change of Hydrogen Sulfide After Traumatic Brain Injury and its Effect in Mice," Neurochemical Research, vol. 38, no. 4, pp. 714725, 2013.

[3] M. Zhang, H. Shan, Y. Wang et al., "The expression changes of cystathionine- $\beta$-synthase in brain cortex after traumatic brain injury," Journal of Molecular Neuroscience, vol. 51, no. 1, pp. 57-67, 2013.

[4] M. Campolo, E. Esposito, A. Ahmad et al., "Hydrogen sulfidereleasing cyclooxygenase inhibitor ATB-346 enhances motor function and reduces cortical lesion volume following traumatic brain injury in mice," J Neuroinflammation, vol. 11, no. 1, p. 196, 2014.

[5] X. Jiang, Y. Huang, W. Lin, D. Gao, and Z. Fei, "Protective effects of hydrogen sulfide in a rat model of traumatic brain injury via activation of mitochondrial adenosine triphosphatesensitive potassium channels and reduction of oxidative stress," The Journal of Surgical Research, vol. 184, no. 2, pp. e27-e35, 2013.

[6] S. A. Karimi, N. Hosseinmardi, M. Janahmadi, M. Sayyah, and R. Hajisoltani, "The protective effect of hydrogen sulfide $\left(\mathrm{H}_{2} \mathrm{~S}\right)$ on traumatic brain injury (TBI) induced memory deficits in rats," Brain Research Bulletin, vol. 134, pp. 177-182, 2017.

[7] K. Xu, F. Wu, K. Xu et al., "NaHS restores mitochondrial function and inhibits autophagy by activating the PI3K/Akt/mTOR signalling pathway to improve functional recovery after traumatic brain injury," Chemico-Biological Interactions, vol. 286, pp. 96-105, 2018.
[8] M. Zhang, H. Shan, P. Chang et al., "Upregulation of 3-MST Relates to Neuronal Autophagy After Traumatic Brain Injury in Mice," Cellular and Molecular Neurobiology, vol. 37, no. 2, pp. 291-302, 2017.

[9] B. Geng, J. Yang, Y. Qi et al., " $\mathrm{H}_{2} \mathrm{~S}$ generated by heart in rat and its effects on cardiac function," Biochemical and Biophysical Research Communications, vol. 313, no. 2, pp. 362-368, 2004.

[10] M. W. Warenycia, L. R. Goodwin, C. G. Benishin et al., “Acute hydrogen sulfide poisoning: Demonstration of selective uptake of sulfide by the brainstem by measurement of brain sulfide levels," Biochemical Pharmacology, vol. 38, no. 6, pp. 973981, 1989.

[11] P. K. Kamat, A. Kalani, and N. Tyagi, "Role of hydrogen sulfide in brain synaptic remodeling," Methods in Enzymology, vol. 555, pp. 207-229, 2015.

[12] K. R. Olson, M. J. Healy, Z. Qin et al., "Hydrogen sulfide as an oxygen sensor in trout gill chemoreceptors," American Journal of Physiology-Regulatory, Integrative and Comparative Physiology, vol. 295, no. 2, pp. R669-R680, 2008.

[13] B. Kim, J. Lee, J. Jang, D. Han, and K.-H. Kim, "Prediction on the Seasonal Behavior of Hydrogen Sulfide Using a Neural Network Model," The Scientific World JOURNAL, vol. 11, 1004 pages, 2011.

[14] M. Whiteman, J. S. Armstrong, S. H. Chu et al., "The novel neuromodulator hydrogen sulfide: an endogenous peroxynitrite 'scavenger'?," Journal of Neurochemistry, vol. 90, no. 3, pp. 765-768, 2004.

[15] L. Siebold, A. Obenaus, and R. Goyal, "Criteria to define mild, moderate, and severe traumatic brain injury in the mouse controlled cortical impact model," Experimental Neurology, vol. 310, pp. 48-57, 2018.

[16] S. Yamamoto, H. S. Levin, and D. S. Prough, "Mild, moderate and severe," Current Opinion in Neurology, vol. 31, no. 6, pp. 672-680, 2018.

[17] C.-C. Chiu, Y.-E. Liao, L.-Y. Yang et al., "Neuroinflammation in animal models of traumatic brain injury," Journal of Neuroscience Methods, vol. 272, pp. 38-49, 2016.

[18] A. Hånell, J. Hedin, F. Clausen, and N. Marklund, "Facilitated Assessment of Tissue Loss Following Traumatic Brain Injury," Frontiers in Neurology, vol. 3, 2012.

[19] P. R. Lees-Haley, P. Green, M. L. Rohling, D. D. Fox, and L. M. Allen 3rd, "The lesion(s) in traumatic brain injury: implications for clinical neuropsychology," Archives of Clinical Neuropsychology, vol. 18, no. 6, pp. 585-594, 2003.

[20] N. D. Osier, J. R. Korpon, and C. E. Dixon, Controlled Cortical Impact Model, F. H. Kobeissy, Ed., Brain Neurotrauma, Boca Raton (FL), 2015.

[21] E. W. Baker, H. A. Kinder, J. M. Hutcheson et al., "Controlled cortical impact severity results in graded cellular, tissue, and functional responses in a piglet traumatic brain injury model," Journal of Neurotrauma, vol. 36, no. 1, pp. 61-73, 2019.

[22] N. Osier and C. E. Dixon, "The Controlled Cortical Impact Model of Experimental Brain Trauma: Overview, Research Applications, and Protocol," Methods in Molecular Biology, vol. 1462, pp. 177-192, 2016.

[23] P. Sellappan, J. Cote, P. A. Kreth et al., "Variability and uncertainty in the rodent controlled cortical impact model of traumatic brain injury," Journal of Neuroscience Methods, vol. 312, pp. 37-42, 2019. 
[24] X. Cao, L. Ding, Z. Z. Xie et al., "A review of hydrogen sulfide synthesis, metabolism, and measurement: is modulation of hydrogen sulfide a novel therapeutic for cancer?," Antioxidants \& Redox Signaling, vol. 31, no. 1, pp. 1-38, 2019.

[25] J. Zhang, H. Shan, L. Tao, and M. Zhang, "Biological Effects of Hydrogen Sulfide and Its Protective Role in Intracerebral Hemorrhage," Journal of Molecular Neuroscience, vol. 70, no. 12, pp. 2020-2030, 2020.

[26] E. Łowicka and J. Bełtowski, "Hydrogen sulfide (H2S) - the third gas of interest for pharmacologists," Pharmacological Reports, vol. 59, no. 1, pp. 4-24, 2007.

[27] N. Shibuya, M. Tanaka, M. Yoshida et al., "3-Mercaptopyruvate Sulfurtransferase Produces Hydrogen Sulfide and Bound Sulfane Sulfur in the Brain," Antioxidants \& Redox Signaling, vol. 11, no. 4, pp. 703-714, 2009.

[28] T. S. Bailey, L. N. Zakharov, and M. D. Pluth, "Understanding hydrogen sulfide storage: probing conditions for sulfide release from hydrodisulfides," Journal of the American Chemical Society, vol. 136, no. 30, pp. 10573-10576, 2014.

[29] M. Ishigami, K. Hiraki, K. Umemura, Y. Ogasawara, K. Ishii, and H. Kimura, "A source of hydrogen sulfide and a mechanism of its release in the brain," Antioxidants \& Redox Signaling, vol. 11, no. 2, pp. 205-214, 2009.

[30] W. Guo, J.-t. Kan, Z.-y. Cheng et al., "Hydrogen sulfide as an endogenous modulator in mitochondria and mitochondria dysfunction," Oxidative Medicine and Cellular Longevity, vol. 2012, Article ID 878052, 9 pages, 2012.

[31] B. V. Nagpure and J. S. Bian, "Interaction of Hydrogen Sulfide with Nitric Oxide in the Cardiovascular System," Oxidative Medicine and Cellular Longevity., vol. 2016, article 6904327, pp. 1-16, 2016.

[32] T. L. Guidotti, "Hydrogen sulfide: advances in understanding human toxicity," International Journal of Toxicology, vol. 29, no. 6, pp. 569-581, 2010.

[33] D. Lozano, G. S. Gonzales-Portillo, S. Acosta et al., "Neuroinflammatory responses to traumatic brain injury: etiology, clinical consequences, and therapeutic opportunities," Neuropsychiatric Disease and Treatment, vol. 11, pp. 97-106, 2015.

[34] S. J. Schimmel, S. Acosta, and D. Lozano, "Neuroinflammation in traumatic brain injury: A chronic response to an acute injury," Brain Circulation, vol. 3, no. 3, pp. 135-142, 2017.

[35] H. A. Seifert and K. R. Pennypacker, "Molecular and Cellular Immune Responses to Ischemic Brain Injury," Translational Stroke Research, vol. 5, no. 5, pp. 543-553, 2014.

[36] Q. Zhang, L. Yuan, D. Liu et al., "Hydrogen sulfide attenuates hypoxia-induced neurotoxicity through inhibiting microglial activation," Pharmacological Research, vol. 84, pp. 32-44, 2014.

[37] N.-1. Xiang, J. Liu, Y.-j. Liao et al., “Abrogating ClC-3 Inhibits LPS-induced Inflammation via Blocking the TLR4/NF- $\kappa \mathrm{B}$ Pathway," Scientific Reports, vol. 6, 2016.

[38] X. Zhou, Y. Cao, G. Ao et al., "CaMKK $\beta$-Dependent Activation of AMP-Activated Protein Kinase Is Critical to Suppressive Effects of Hydrogen Sulfide on Neuroinflammation," Antioxidants \& Redox Signaling, vol. 21, no. 12, pp. 17411758, 2014.

[39] L. Li, M. Bhatia, Y. Z. Zhu et al., "Hydrogen sulfide is a novel mediator of lipopolysaccharide-induced inflammation in the mouse," The FASEB Journal, vol. 19, no. 9, pp. 1196-1198, 2005.
[40] J. Zhang, S. W. S. Sio, S. Moochhala, and M. Bhatia, "Role of Hydrogen Sulfide in Severe Burn Injury-Induced Inflammation in Mice," Molecular Medicine, vol. 16, pp. 417-424, 2010.

[41] E. Tönnies and E. Trushina, "Oxidative Stress, Synaptic Dysfunction, and Alzheimer's Disease," Journal of Alzheimer's Disease, vol. 57, no. 4, pp. 1105-1121, 2017.

[42] Y. Kimura, Y.-I. Goto, and H. Kimura, "Hydrogen Sulfide Increases Glutathione Production and Suppresses Oxidative Stress in Mitochondria," Antioxidants \& Redox Signaling, vol. 12, no. 1, pp. 1-13, 2010.

[43] Y. Kimura and H. Kimura, "Hydrogen sulfide protects neurons from oxidative stress," The FASEB Journal, vol. 18, no. 10, pp. 1165-1167, 2004.

[44] O. W. Griffith, "Biologic and pharmacologic regulation of mammalian glutathione synthesis," Free Radical Biology \& Medicine, vol. 27, no. 9-10, pp. 922-935, 1999.

[45] J.-i. Sagara, K. Miura, and S. Bannai, "Cystine Uptake and Glutathione Level in Fetal Brain Cells in Primary Culture and in Suspension," Journal of Neurochemistry, vol. 61, no. 5, pp. 1667-1671, 1993.

[46] T. H. Murphy, M. Miyamoto, A. Sastre, R. L. Schnaar, and J. T. Coyle, "Glutamate toxicity in a neuronal cell line involves inhibition of cystine transport leading to oxidative stress," Neuron, vol. 2, no. 6, pp. 1547-1558, 1989.

[47] T. Corsello, N. Komaravelli, and A. Casola, "Role of hydrogen sulfide in NRF2- assssssnd sirtuin-dependent maintenance of cellular redox balance," Antioxidants, vol. 7, no. 10, p. 129, 2018.

[48] S. Zhao, T. Song, Y. Gu et al., "Hydrogen sulfide alleviates liver injury via S-sulfhydrated-Keap1/Nrf2/LRP1 pathway," Нераtology, 2020.

[49] C. Szabó, "Hydrogen sulphide and its therapeutic potential," Nature Reviews Drug Discovery, vol. 6, no. 11, pp. 917-935, 2007.

[50] Q. Xiao, J. Ying, L. Xiang, and C. Zhang, "The biologic effect of hydrogen sulfide and its function in various diseases," Medicine, vol. 97, no. 44, p. e13065, 2018.

[51] J. Zhang, C. Shi, H. Wang et al., "Hydrogen sulfide protects against cell damage through modulation of PI3K/Akt/Nrf2 signaling," The International Journal of Biochemistry \& Cell Biology, vol. 117, p. 105636, 2019.

[52] B. M. Polster, K. W. Kinnally, and G. Fiskum, "BH3 death domain peptide induces cell type-selective mitochondrial outer membrane permeability," Journal of Biological Chemistry, vol. 276, pp. 37887-37894, 2001.

[53] Y. Luo, X. Liu, Q. Zheng et al., "Hydrogen sulfide prevents hypoxia-induced apoptosis via inhibition of an $\mathrm{H}_{2} \mathrm{O}_{2}$-activated calcium signaling pathway in mouse hippocampal neurons," Biochemical and Biophysical Research Communications, vol. 425, no. 2, pp. 473-477, 2012.

[54] Y. Luo, X. Yang, S. Zhao et al., "Hydrogen sulfide prevents OGD/R-induced apoptosis via improving mitochondrial dysfunction and suppressing an ROS-mediated caspase-3 pathway in cortical neurons," Neurochemistry International, vol. 63, no. 8, pp. 826-831, 2013.

[55] K. Ji, L. Xue, J. Cheng, and Y. Bai, "Preconditioning of $\mathrm{H}_{2} \mathrm{~S}$ inhalation protects against cerebral ischemia/reperfusion injury by induction of HSP70 through PI3K/Akt/Nrf2 pathway," Brain Research Bulletin, vol. 121, pp. 68-74, 2016.

[56] M.-y. Hong, J.-z. Cui, R. Li et al., "Effect of expression of c-jun $\mathrm{N}$-terminal kinase on neuron autophagy following diffuse 
brain injury in rats," Zhonghua Wai Ke Za Zhi, vol. 50, no. 2, pp. 166-170, 2012.

[57] N. Sen, B. D. Paul, M. M. Gadalla et al., "Hydrogen SulfideLinked Sulfhydration of NF- $\kappa$ B Mediates Its Antiapoptotic Actions," Molecular Cell, vol. 45, no. 1, pp. 13-24, 2012.

[58] J. Wu and M. M. Lipinski, "Autophagy in Neurotrauma: Good, Bad, or Dysregulated," Cells, vol. 8, no. 7, p. 693, 2019.

[59] C. Sarkar, Z. Zhao, S. Aungst, B. Sabirzhanov, A. I. Faden, and M. M. Lipinski, "Impaired autophagy flux is associated with neuronal cell death after traumatic brain injury," Autophagy, vol. 10, no. 12, pp. 2208-2222, 2015.

[60] S. Bialik, S. K. Dasari, and A. Kimchi, "Autophagy-dependent cell death - where, how and why a cell eats itself to death," Journal of Cell Science, vol. 131, no. 18, p. jcs215152, 2018.

[61] D. Denton and S. Kumar, "Autophagy-dependent cell death," Cell Death \& Differentiation, vol. 26, no. 4, pp. 605-616, 2019.

[62] L. Zhang and H. Wang, "Autophagy in Traumatic Brain Injury: A New Target for Therapeutic Intervention," Frontiers in Molecular Neuroscience, vol. 11, 2018.

[63] C. Gao, P. Chang, L. Yang et al., "Neuroprotective effects of hydrogen sulfide on sodium azide-induced oxidative stress in PC12 cells," International Journal of Molecular Medicine, vol. 41, pp. 242-250, 2017.

[64] J.-F. Wang, Y. Li, J.-N. Song, and H.-G. Pang, "Role of hydrogen sulfide in secondary neuronal injury," Neurochemistry International, vol. 64, pp. 37-47, 2014.

[65] W. Zhao, "The vasorelaxant effect of H2S as a novel endogenous gaseous KATP channel opener," The EMBO Journal, vol. 20, no. 21, pp. 6008-6016, 2001.

[66] C. W. Leffler, H. Parfenova, S. Basuroy, J. H. Jaggar, E. S. Umstot, and A. L. Fedinec, "Hydrogen sulfide and cerebral microvascular tone in newborn pigs," American Journal of Physiology-Heart and Circulatory Physiology, vol. 300, no. 2, pp. H440-H447, 2011.

[67] C.-X. Liu, Y.-R. Tan, Y. Xiang, C. Liu, X.-A. Liu, and X.Q. Qin, "Hydrogen Sulfide Protects against Chemical Hypoxia-Induced Injury via Attenuation of ROS-Mediated $\mathrm{Ca} 2+$ Overload and Mitochondrial Dysfunction in Human Bronchial Epithelial Cells," BioMed Research International, vol. 2018, 9 pages, 2018.

[68] M. A. García-Bereguiaín, A. K. Samhan-Arias, F. J. MartínRomero, and C. Gutiérrez-Merino, "Hydrogen sulfide raises cytosolic calcium in neurons through activation of L-type Ca2+ channels," Antioxid Redox Signal, vol. 10, no. 1, pp. 31-42, 2008.

[69] Q. C. Yong, C. H. Choo, B. H. Tan, C.-M. Low, and J.-S. Bian, "Effect of hydrogen sulfide on intracellular calcium homeostasis in neuronal cells," Neurochemistry International, vol. 56, no. 3, pp. 508-515, 2010.

[70] Y. Nagai, M. Tsugane, J.-I. Oka, and H. Kimura, "Hydrogen sulfide induces calcium waves in astrocytes," The FASEB Journal, vol. 18, no. 3, pp. 557-559, 2004.

[71] W. J. Cahill, J. H. Calvert, and J. H. Zhang, "Mechanisms of early brain injury after subarachnoid hemorrhage," Journal of Cerebral Blood Flow \& Metabolism, vol. 26, no. 11, pp. 13411353, 2006.

[72] S. Cao, P. Zhu, X. Yu et al., "Hydrogen sulfide attenuates brain edema in early brain injury after subarachnoid hemorrhage in rats: Possible involvement of MMP-9 induced blood- brain barrier disruption and AQP4 expression," Neuroscience Letters, vol. 621, pp. 88-97, 2016.
[73] T. Dóczi, “The pathogenetic and prognostic significance of blood-brain barrier damage at the acute stage of aneurysmal subarachnoid haemorrhage. Clinical and experimental studies," Acta Neurochirurgica, vol. 77, pp. 110-132, 1985.

[74] K. Schöller, A. Trinkl, M. Klopotowski et al., "Characterization of microvascular basal lamina damage and blood-brain barrier dysfunction following subarachnoid hemorrhage in rats," Brain Research, vol. 1142, pp. 237-246, 2007.

[75] G. T. Manley, M. Fujimura, T. Ma et al., “Aquaporin-4 deletion in mice reduces brain edema after acute water intoxication and ischemic stroke," Nature Medicine, vol. 6, no. 2, pp. 159$163,2000$.

[76] F. Zhao, J. Deng, X. Xu et al., “Aquaporin-4 deletion ameliorates hypoglycemia-induced $\mathrm{BBB}$ permeability by inhibiting inflammatory responses," Journal of Neuroinflammation, vol. 15, no. 1, p. 157, 2018.

[77] H. Kimura, "Hydrogen Sulfide Induces Cyclic AMP and Modulates the NMDA Receptor," Biochemical and Biophysical Research Communications, vol. 267, no. 1, pp. 129-133, 2000.

[78] A. Stein and S. M. Bailey, "Redox biology of hydrogen sulfide: Implications for physiology, pathophysiology, and pharmacology," Redox Biology, vol. 1, no. 1, pp. 32-39, 2013.

[79] J.-y. Zhang, Y.-p. Ding, Z. Wang, Y. Kong, R. Gao, and G. Chen, "Hydrogen sulfide therapy in brain diseases: from bench to bedside," Medical Gas Research, vol. 7, no. 2, pp. 113-119, 2017. 